\title{
Preoperative Serum Leptin Level Is Associated with Preoperative Pain Threshold and Postoperative Analgesic Consumption in Patients Undergoing Cesarean Section
}

\author{
Nurten Seringec Akkececi ${ }^{\mathrm{a}}$ Gozen Oksuz ${ }^{\mathrm{b}}$ Aykut Urfalioğlu ${ }^{\mathrm{b}}$ \\ Ramazan Gunesacar ${ }^{c}$ Murat Bakacak $^{d}$ Mahmut Arslan $^{b}$ \\ Bekir Mehmet Kellecic \\ a Department of Physiology, Faculty of Medicine, Kahramanmaras Sutcu Imam University, Kahramanmaras,

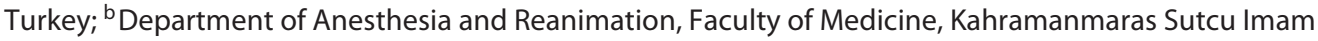 \\ University, Kahramanmaras, Turkey; ${ }^{\complement}$ Department of Medical Biology, Faculty of Medicine, Kahramanmaras Sutcu \\ Imam University, Kahramanmaras, Turkey; ${ }^{\text {d} D e p a r t m e n t ~ o f ~ O b s t e t r i c s ~ a n d ~ G y n e c o l o g y, ~ F a c u l t y ~ o f ~ M e d i c i n e, ~}$ \\ Kahramanmaras Sutcu Imam University, Kahramanmaras, Turkey
}

\section{Significance of the Study}

- Preoperative serum leptin was found to be associated with preoperative pain threshold and postoperative analgesic consumption. Subjects with higher serum leptin levels had reduced pain threshold and increased analgesic consumption. The serum leptin level prior to cesarean section may be useful in predicting postoperative analgesic requirement patients and help improve strategies for postoperative pain management.

\section{Keywords}

Leptin - Labor pain · Pain threshold · Postoperative analgesic consumption · Visual analog scale

\footnotetext{
Abstract

Objective: This study aimed to investigate the preoperative level of serum leptin in cesarean section (C-section) patients with and without acute labor pain and its association with postoperative analgesic consumption and preoperative pain threshold. Materials and Methods: Preoperative leptin levels, preoperative pain threshold, postoperative analgesic consumption in the first $24 \mathrm{~h}$, and postoperative pain sever-
}

ity (visual analog scale (VAS) scores at 1, 2, 4, 6, 12, and $24 \mathrm{~h}$ postoperatively) in C-section patients with labor pain (emergency C-section; $n=21$ ) and without labor pain (elective Csection; $n=25$ ) were compared. Results: There were no significant differences between the groups regarding the demographic characteristics. Leptin levels, postoperative VAS scores, and analgesic consumption were significantly higher in the group with labor pain, while the preoperative pain threshold was lower. Serum leptin levels correlated negatively with pain threshold and positively with postoperative analgesic consumption. Multiple linear regression analyses in our study revealed that the preoperative leptin levels and having an emergency $C$-section independently af-

\begin{tabular}{ll}
\hline KARGER & $\begin{array}{l}\text { ( 2019 The Author(s) Karger } \\
\text { Published by S. Karger AG, Basel Open cacess }\end{array}$ \\
E-Mail karger@karger.com & $\begin{array}{l}\text { This is an Open Access article licensed under the Creative Commons } \\
\text { Attribution-NonCommercial-4.0 International License (CC BY-NC) } \\
\text { (http://www.karger.com/Services/OpenAccessLicense), applicable to } \\
\text { the online version of the article only. Usage and distribution for com- } \\
\text { mercial purposes requires written permission. }\end{array}$
\end{tabular}

Nurten Seringec Akkececi

Department of Physiology, Faculty of Medicine

Kahramanmaras Sutcu Imam University, Bahcelievler Campus, Emniyet Street

TR-46100 Kahramanmaras (Turkey)

E-Mail seringec@ hotmail.com 
fected the postoperative analgesic consumption and preoperative pain threshold, whereas their combined effects on these parameters were statistically not significant. Conclusion: Preoperative levels of serum leptin were higher in Csection patients with labor pain than in those without labor pain, and increased serum leptin levels were associated with decreased preoperative pain threshold and increased postoperative analgesic consumption in our study population. Postoperative analgesic requirements may vary among patients, and their requirements might be predicted using preoperative indicators. Serum levels of leptin might be one such indicator and this warrants further studies with larger sample sizes.

(C) 2019 The Author(s)

Published by S. Karger AG, Basel

\section{Introduction}

Leptin is a peptide hormone, which is encoded by the obese $(o b)$ gene, contains 167 amino acids, and is secreted by various tissues, mainly the adipose tissue. Several studies have shown the central and peripheral effects of leptin on food intake and energy metabolism [1] and inflammation [2]. Atawi et al. [3] showed that serum levels of leptin were higher in pregnant women than in nonpregnant women. During pregnancy, circulating leptin concentrations rise during the first and second trimesters and peak in the third trimester followed by a drastic decrease after delivery to return to normal concentration in 6 weeks [4]. In addition to adipose tissue production, increased levels of leptin in pregnant women have been reported to result from placental production [5]. It has been reported that the mode of delivery and duration of labor are significantly associated with leptin [6]. Logan et al. [6] showed that levels of leptin were lower in women undergoing elective cesarean delivery than in those undergoing emergency cesarean delivery and the vaginal delivery group. Yoshimitsu et al. [7] demonstrated that placental release of leptin increased during labor and that arterial and umbilical venous levels of leptin in women who had vaginal deliveries were higher than in those who had elective cesarean deliveries. However, it remains unclear why leptin levels increase during labor and why leptin levels differ based on the mode of delivery.

Recent studies have indicated that leptin has a role in the modulation of pain, but the mechanism of this remains unclear. Hu et al. [8] demonstrated that leptin is involved in nociception. Intrathecal administration of leptin to rats induced mechanical allodynia and thermal hyperalgesia [9]. There are reports indicating that leptin levels were increased in chronic pain conditions and positively correlated with pain severity [10-12]. Younger et al. [13] also suggested a link between higher levels of leptin and greater daily pain. Plasma leptin was shown to have a half-life of approximately $30 \mathrm{~min}$ and to reach physiological concentrations within a few hours, which suggests that regulation of its levels could be associated with short-term events and that it could be involved in the regulation of short-term events [14].

Post-cesarean section (C-section) pain is one of the most important acute pain complaints for women. After a C-section, almost 1 in 5 patients will experience severe acute pain [15]. The pain can be felt and described differently, and some patients experience more severe pain than others after a C-section even when the same type of anesthesia is applied. We hypothesized that this difference might be related to increased preoperative levels of serum leptin in acute labor pain and that leptin levels could lead to increased use of analgesics in the postoperative period. We also hypothesized that the patients could have altered preoperative pain threshold and report higher pain scores after the operation due to hyperalgesia induced by high levels of leptin.

This study aimed to investigate the preoperative level of serum leptin in patients who underwent $\mathrm{C}$-section with and without acute labor pain, and to ascertain whether there was an association between preoperative levels of leptin and postoperative use of analgesics. The preoperative pain threshold and postoperative pain severity of patients were also investigated.

\section{Materials and Methods}

\section{Subjects}

Our patients were divided into two groups as follows: the labor pain group (LPG, pregnant women who had an emergency C-section with labor pain) and the no pain group (NPG, pregnant women who were scheduled to undergo elective C-section without labor pain). Patients with 3 or more regular uterine contractions on cardiotocographic evaluation in $20 \mathrm{~min}$ or uterine performance of $>120$ Montevideo units in 20 min were defined as having labor pain. Of a total of 46 pregnant women in the study, 21 were in the LPG while 25 were in the NPG. Both groups received spinal anesthesia for $\mathrm{C}$-section. Exclusion criteria were being under the age of 18 , having an abnormal pregnancy with preeclampsia, eclampsia, gestational diabetes, or hypertension, having a comorbid disease, systemic disease, cardiovascular disease, diabetes, obesity, or infection, or currently undergoing any medical treatment.

American Society of Anesthesiologists (ASA) score, age, weight, height, body mass index (BMI), gestational week at delivery, and the number of previous $\mathrm{C}$-sections of the patients were recorded. 
Table 1. Demographic, preoperative, and postoperative characteristics of the patients

\begin{tabular}{|c|c|c|c|c|c|}
\hline & \multicolumn{2}{|c|}{ No labor pain group } & \multicolumn{2}{|c|}{ Labor pain group } & \multirow[t]{2}{*}{$p$} \\
\hline & mean $\pm \mathrm{SD}$ & median [IQR] & mean $\pm S D$ & median [IQR] & \\
\hline ASA score & $2.04 \pm 0.2$ & $2[2-2]$ & $2 \pm 0$ & $2[2-2]$ & $0.383^{*}$ \\
\hline Age, years & $30.6 \pm 6.01$ & $30[27-35]$ & $27.95 \pm 7.14$ & $29[22-34]$ & $0.179^{* *}$ \\
\hline Weight, kg & $78.44 \pm 12.44$ & $78[69-89]$ & $77.33 \pm 13.56$ & $76[66-85]$ & $0.774^{* *}$ \\
\hline Height, cm & $162.56 \pm 4.31$ & $163[160-165]$ & $162.24 \pm 6.79$ & $162[160-165]$ & $0.846^{* *}$ \\
\hline $\mathrm{BMI}, \mathrm{kg} / \mathrm{m}^{2}$ & $29.54 \pm 4.18$ & $29.9[26.9-32.2]$ & $29.22 \pm 3.79$ & $28.9[26.4-31.14]$ & $0.789^{* *}$ \\
\hline Gestational week at delivery & $38.56 \pm 0.58$ & 39 [38-39] & $38.95 \pm 0.67$ & 39 [39-39] & $0.060 *$ \\
\hline Cesarian sections, $n$ & $2.52 \pm 1$ & $2[2-3]$ & $2.24 \pm 1$ & $2[2-3]$ & $0.346^{*}$ \\
\hline Leptin, ng/mL & $5.7 \pm 2.24$ & $5.28[3.55-7.58]$ & $10.33 \pm 6.51$ & $8.95[4.47-14.26]$ & $0.010^{*}$ \\
\hline Pain threshold & $13.7 \pm 0.81$ & $13.6[13-14]$ & $12.7 \pm 0.59$ & $12.6[12.3-13]$ & $<0.001^{*}$ \\
\hline Analgesic consumption, mg/24 h & $145.88 \pm 28.11$ & $153[135-170]$ & $212.95 \pm 42.38$ & $198[180-225]$ & $<0.001^{*}$ \\
\hline VAS1 & $2.84 \pm 1.25$ & $2[2-4]$ & $4.05 \pm 1.24$ & $4[3-4]$ & 0.003* \\
\hline VAS2 & $2.2 \pm 1.19$ & $2[1-3]$ & $3.19 \pm 0.81$ & $3[3-4]$ & $0.004^{*}$ \\
\hline VAS4 & $1.68 \pm 0.75$ & $2[1-2]$ & $2.29 \pm 0.85$ & $2[2-3]$ & $0.017^{*}$ \\
\hline VAS6 & $1.56 \pm 0.71$ & $1[1-2]$ & $2.1 \pm 0.77$ & $2[2-2]$ & $0.017^{*}$ \\
\hline VAS12 & $1.44 \pm 0.58$ & $1[1-2]$ & $1.86 \pm 0.57$ & $2[2-2]$ & $0.017^{*}$ \\
\hline VAS24 & $1.44 \pm 0.58$ & $1[1-2]$ & $1.76 \pm 0.54$ & $2[1-2]$ & $0.047^{*}$ \\
\hline
\end{tabular}

SD, standard deviation; IQR, interquartile range; ASA, American Society of Anesthesiologists; BMI, body mass index; VAS, Visual Analog Scale at $1,2,4,6,12$, and 24 h postoperatively. ${ }^{*}$ Mann-Whitney $\mathrm{U}$ test, ${ }^{* *}$ Independent samples $t$ test.

\section{Measurement of Serum Leptin}

For serum leptin measurements, blood was drawn in the preoperative waiting room prior to the application of spinal anesthesia. The blood samples were centrifuged, and serum was kept at $-20^{\circ} \mathrm{C}$ until processing. Serum leptin levels were determined by the use of a direct Sandwich ELISA kit (DRG Instruments GmbH, Marburg, Germany, Cat No: EIA-2395).

\section{Measurement Pain Threshold}

Manual dolorimetry was used to assess the pain threshold in the nondominant hand of the patients in the preoperative waiting room prior to spinal anesthesia. The head of the dolorimeter was positioned vertically at the wrist of the nondominant hand. Pressure was applied in increments of $1 \mathrm{~kg} / \mathrm{cm}^{2} / \mathrm{s}$ and was stopped when the pain was first perceived; the pressure applied was recorded in $\mathrm{kg} / \mathrm{cm}^{2}$. The measurement was repeated three times for each patient. The mean of three measurements was recorded as the pain threshold value.

\section{Postoperative Pain Severity on the Visual Analog Scale}

All patients were asked to report any pain through a self-assessment instrument: the visual analog scale (VAS). Patients were informed about VAS and its pain scoring system, which ranges from 0 (no pain) to 10 (extreme pain). All patients were monitored carefully, and their VAS scores were recorded at 1, 2, 4, 6, 12, and $24 \mathrm{~h}$ postoperatively.

\section{Consumption of Postoperative Analgesic}

For postoperative analgesia, both groups of patients received intravenous tramadol through a patient-controlled analgesia pump for $24 \mathrm{~h}$. The patients were informed about the patient-controlled analgesia device and instructed to use it when they experienced pain with initial settings for intravenous tramadol at a bolus dose of $9 \mathrm{mg}$ $(2 \mathrm{~mL})$, lockout time of $20 \mathrm{~min}$, and hour limit dosage of $27 \mathrm{mg}$.

\section{Estimation of Sample Size}

Estimation of sample size was done using the G* Power3 Analysis Program (Heinrich Heine University, Düsseldorf, Germany). A pilot study was conducted on 10 subjects from each group. The power analysis was performed based on the mean analgesic use of the patients in $24 \mathrm{~h}$, which was $154 \pm 28.3 \mathrm{mg} / 24 \mathrm{~h}$ in the NPG and $190 \pm 40.2 \mathrm{mg} / 24 \mathrm{~h}$ in the LPG. The sample size was calculated at a power of $90 \%$ and a significance level of $5 \%$; it was determined that approximately 21 patients per group would be necessary to obtain statistically significant values. To prevent statistical bias, the data collection and data analysis teams were kept separate.

\section{Statistics}

The data were summarized as mean \pm standard deviation and/ or median [interquartile range] for continuous variables. Normal distribution of numerical variables was checked with the Kolmogorov-Smirnov test. For the comparison of two independent groups, the independent samples $t$ test was used when the data was normally distributed and Mann-Whitney U test when it was not. Spearman's rho correlation coefficient was used to examine the relationships between leptin, pain threshold, VAS score, and analgesic consumption. Multiple regression analysis was performed to examine the effects of leptin levels and group variables on analgesic consumption and pain threshold. Statistical analyses were performed with Jamovi (version 0.9.5.12, retrieved from https://www. jamovi.org) and SPSS 16.0 for Windows (SPSS Inc., Chicago, IL, USA). $p<0.05$ was considered to be statistically significant. 
Table 2. Correlation between leptin level and postoperative analgesic consumption or preoperative pain threshold

\begin{tabular}{lllcc}
\hline Data set & & Spearman's rho & $p$ \\
\hline Whole study group & Leptin & Analgesic consumption & 0.504 & $<\mathbf{0 . 0 0 1}$ \\
& Leptin & Pain threshold & -0.527 & $<\mathbf{0 . 0 0 1}$ \\
\hline No labor pain group & Leptin & Analgesic consumption & 0.230 & 0.269 \\
& Leptin & Pain threshold & -0.263 & 0.205 \\
\hline Labor pain group & Leptin & Analgesic consumption & 0.551 & $\mathbf{0 . 0 1 0}$ \\
& Leptin & Pain threshold & -0.550 & $\mathbf{0 . 0 1 0}$ \\
\hline
\end{tabular}

Table 3. Correlation between preoperative pain threshold and postoperative VAS scores

\begin{tabular}{lllr}
\hline Data set & Pain threshold & Spearman's rho & \multicolumn{1}{l}{$p$} \\
\hline \multirow{2}{*}{ Whole study group } & VAS1 & -0.682 & $<\mathbf{0 . 0 0 1}$ \\
& VAS2 & -0.680 & $<\mathbf{0 . 0 0 1}$ \\
& VAS4 & -0.542 & $<\mathbf{0 . 0 0 1}$ \\
& VAS6 & -0.523 & $<\mathbf{0 . 0 0 1}$ \\
& VAS12 & -0.509 & $<\mathbf{0 . 0 0 1}$ \\
& VAS24 & -0.449 & $\mathbf{0 . 0 0 2}$ \\
\hline No labor pain group & VAS1 & -0.642 & $<\mathbf{0 . 0 0 1}$ \\
& VAS2 & -0.635 & $<\mathbf{0 . 0 0 1}$ \\
& VAS4 & -0.661 & $<\mathbf{0 . 0 0 1}$ \\
& VAS6 & -0.604 & $\mathbf{0 . 0 0 1}$ \\
& VAS12 & -0.619 & $<\mathbf{0 . 0 0 1}$ \\
& VAS24 & -0.619 & $<\mathbf{0 . 0 0 1}$ \\
\hline Labor pain group & VAS1 & -0.321 & 0.155 \\
& VAS2 & -0.292 & 0.199 \\
& VAS4 & 0.000 & 1.000 \\
& VAS6 & -0.014 & 0.953 \\
& VAS12 & 0.075 & 0.747 \\
VAS24 & 0.155 & 0.501 \\
\hline
\end{tabular}

VAS, Visual Analog Scale at 1,2,4,6,12, and 24 h postoperatively.

\section{Results}

\section{Descriptive Data}

Demographic characteristics, leptin levels, preoperative pain threshold, and postoperative characteristics of the patients in the NPG and LPG are presented in Table 1. No significant differences were found between the groups with respect to ASA score, age, weight, height, $\mathrm{BMI}$, gestational week at delivery, and the number of previous $\mathrm{C}$-sections $(p>0.05)$. Leptin levels in the LPG were higher than those in the NPG $(5.28 \mathrm{ng} / \mathrm{mL}$ [3.55-7.58]) $(p<0.05)$. The postoperative analgesic consumption in the first 24 hours after $\mathrm{C}$-section was significantly higher in the LPG than in the NPG $(p<0.05)$. The postoperative VAS scores at $1,2,4,6,12$, and $24 \mathrm{~h}$ postoperatively were significantly higher in the LPG than the NPG $(p<0.05)$. On the other hand, preoperative pain threshold was significantly lower in the LPG compared to the NPG ( $p<$ 0.05).

\section{Correlation of Leptin and Analgesic Use}

Correlation coefficients of the relationships between leptin level and postoperative analgesic consumption or preoperative pain threshold are given in Table 2. In the whole study group and in the LPG, there was a significant positive correlation between leptin level and postoperative analgesic consumption $(p<0.001)$ and a significant negative correlation between leptin level and preoperative pain threshold $(p<0.001)$. In the NPG, no correlation was found between these variables. Leptin levels were also positively correlated with the BMI $(r=0.338, p=0.022)$.

\section{Correlation of Pain Threshold and Pain Scores}

Correlation coefficients of the relationships between preoperative pain threshold and postoperative pain scores are depicted in Table 3. In the whole study group and in the NPG, there was a significant negative correlation between preoperative pain threshold and pain scores at 1,2 , $4,6,12$, and $24 \mathrm{~h}$ after the operation $(p<0.05$ for all). In the LPG, no correlation was found between these variables.

\section{Multiple Linear Regression Analyses}

Multiple linear regression analysis revealed that the preoperative leptin levels and having an emergency Csection independently affected the postoperative analgesic consumption ( $p=0.038$ and $p=0.019$, respectively) 
Table 4. Multiple linear regression analysis of the association between analgesic consumption and leptin level

\begin{tabular}{|c|c|c|c|c|c|}
\hline Constant & $152.63(129.69$ to 175.57$)$ & 11.37 & NA & 13.43 & $<0.001$ \\
\hline Leptin & $3.42(0.20$ to 6.63$)$ & 1.59 & 0.37 & 2.15 & 0.038 \\
\hline Group (Labor pain - No pain group) & $55.49(9.60$ to 101.37$)$ & 22.74 & 0.58 & 2.44 & 0.019 \\
\hline Leptin $\times$ group (Labor pain $\times$ No pain group) & $-0.53(-6.96$ to 5.90$)$ & 3.19 & -0.06 & -0.167 & 0.868 \\
\hline
\end{tabular}

Dependent variable: analgesic consumption. NA, not applicable; SE, standard error. $R^{2}: 0.578$, Model test: $p<0.001$.

Table 5. Multiple linear regression analysis of the association between pain threshold and leptin level

\begin{tabular}{|c|c|c|c|c|c|}
\hline Predictor variables & $\begin{array}{l}\text { Unstandardized coefficient } \beta \\
(95 \% \mathrm{CI})\end{array}$ & SE & $\begin{array}{l}\text { Standardized } \\
\text { coefficients }\end{array}$ & $t$ & $p$ \\
\hline Constant & $13.73(13.25$ to 14.21$)$ & 0.24 & NA & 57.53 & $<0.001$ \\
\hline Leptin & $-0.07(-0.14$ to -0.01$)$ & 0.03 & -0.44 & -2.19 & 0.034 \\
\hline Group (Labor pain - No pain group) & $-1.06(-2.03$ to -0.10$)$ & 0.48 & 0.61 & -2.23 & 0.031 \\
\hline Leptin $\times$ group (Labor pain $\times$ No pain group) & $0.05(-0.08$ to 0.19$)$ & 0.07 & 0.30 & 0.76 & 0.454 \\
\hline
\end{tabular}

Dependent variable: pain threshold. NA, not applicable; SE, standard error. $R^{2}: 0.422$, Model test: $p<0.001$.

(Table 4). That is, the analgesics usage increased as the patient's preoperative leptin level increased or if the patient had undergone emergency $\mathrm{C}$-section. However, this effect was not synergistic $(p=0.868)$. Similarly, the preoperative leptin levels and having had an emergency Csection independently affected the preoperative pain threshold ( $p=0.034$ and $p=0.031$, respectively) (Table $5)$. In other words, the pain threshold decreased as the patient's preoperative leptin level increased or if the patient had undergone emergency $\mathrm{C}$-section, although their combined effect was not significant $(p=0.454)$ (Table 5).

\section{Discussion}

Very few studies have investigated the relationship between preoperative levels of serum leptin and the severity of postoperative pain, postoperative analgesic consumption, or preoperative pain threshold in humans. Our study showed that preoperative levels of serum leptin, postoperative analgesic consumption, and all postoperative VAS scores were significantly higher in patients undergoing C-section with labor pain (LPG) than in those without labor pain (NPG), whereas preoperative pain

Leptin Levels, Pain Threshold, and Analgesic Consumption threshold was significantly lower in the former group. In accordance with these findings, the correlation analyses indicated that preoperative levels of leptin correlated positively with postoperative analgesic consumption and negatively with preoperative pain threshold in the LPG or in the whole study sample. Interestingly, they were not correlated in the NPG. On the other hand, preoperative pain threshold correlated negatively with postoperative VAS scores at all time points in the NPG or in the whole study sample but not in the LPG. Multiple linear regression analyses in our study revealed that preoperative levels of leptin and having an emergency $\mathrm{C}$-section independently affected postoperative analgesic consumption and preoperative pain threshold, whereas their combined effect on postoperative analgesic consumption and pain threshold were statistically not significant.

Recent studies have suggested a role for leptin in the modulation of pain, but the mechanism is not well understood. An animal study demonstrated that leptin was involved in the development of allodynia and exacerbation of neuropathic pain [16]. Other studies conducted on animals have shown that leptin mediates nociceptive behavior [9] and alters nociceptive response [17]. It has been reported that leptin increases pain sensitivity $[18,19]$, and 
leptin-deficient mice have low pain sensitivity [16] although the mechanism of this effect remains unclear. Leptin may increase pain sensitivity perhaps via enhancing N-methyl-D-aspartate receptor function [18] or increasing alpha-melanocyte-stimulating hormone synthesis [20], which have been reported to increase pain sensitivity [21]. However, not much is known about the pain-related effects of leptin in humans.

Previous studies on humans have reported that serum leptin concentration increased in chronic pain condition such as osteoarthritis [12], fibromyalgia [22] and migraine [23]. Furthermore, Bedaiwy et al. [11] showed that the concentration of leptin in the peritoneal fluid was higher in patients with endometriosis. They also found that the leptin concentration in peritoneal fluid positively correlated with chronic pelvic pain. Gandhi et al. [10] reported that the leptin concentration in shoulder synovial fluid positively correlated with shoulder pain in osteoarthritis patients.

Labor is one of the most important acute pain conditions for women [24]. In the present study, serum leptin levels were found to be higher in C-section patients with labor pain compared to those without labor pain, which suggests that the level of leptin, which is known to increase in chronic pain conditions, may also be associated with acute labor pain. We also demonstrated that postoperative analgesic consumption and all postoperative VAS scores were significantly higher in the LPG than in the NPG. Our results are in agreement with previous studies on animals and humans that suggested a correlation between increased leptin and acute or chronic pain severity $[10,11,18]$. In contrast, a retrospective study by Carvalho and colleagues [25] found no significant difference between women who underwent unplanned (emergency) cesarean delivery under epidural anesthesia and those with scheduled cesarean delivery under spinal anesthesia in terms of postoperative pain and total opioid consumption. That study did not include an analysis of leptin levels in their patients but concluded that the two groups, which correspond to the LPG and NPG in our study, might be comparable in terms of postoperative pain and opioid consumption. However, the two groups in that study had different methods of anesthesia, and epidural anesthesia starts later and acts longer than spinal anesthesia, which might have affected the postoperative pain and analgesic consumption.

A few animal studies have investigated the association between leptin and pain threshold. Kutlu et al. [19] reported that intraperitoneal injection of leptin decreases pain threshold in mice. The relationship between leptin levels and pain threshold in humans has not been explored previously. Our study indicates that increased levels of serum leptin might have caused a decrease in pain threshold. In accordance with this finding, leptin was negatively correlated with pain threshold in the whole study group although not in the NPG. Our study also demonstrated that pain threshold was negatively correlated with all postoperative VAS scores in the whole study group although not in the LPG. However, the postoperative VAS scores in our study were obtained while the patient was under an analgesic regimen, which might have influenced these scores. Nonetheless, these findings are in agreement with those of Buhagiar et al. [26] who reported a significant negative correlation between preoperative electrical pain threshold and post-cesarean pain scores or postoperative paracetamol consumption in patients who were scheduled for elective C-section.

Recently, there have been an increasing number of studies investigating the factors that influence the severity of pain felt by women after C-section, which mainly address the preoperative factors affecting postoperative pain and analgesic consumption after C-section [26-28]. One such study found that postoperative pain scores after $\mathrm{C}$-section were higher in women with preoperative scar hyperalgesia than in those without hyperalgesia, although no significant difference was found between the groups in terms of postoperative analgesic consumption [27]. Another study used a psychological test (State-Trait Anxiety Inventory - STAI) to measure preoperative state and trait anxiety of the patients and demonstrated that preoperative STAI scores correlated with postoperative analgesic consumption [28]. Kurdemir and Özorak [29] suggested that the dose of required postoperative analgesics should be planned preoperatively based on the physical and psychological state of the patients. Our findings also suggest that the postoperative analgesic requirements may vary among patients and that their requirements might be predicted using preoperative indicators; serum leptin levels could be one such indicator, and the patient's postoperative analgesic regimen might be planned accordingly.

It is well known that serum leptin level varies depending on demographical characteristics such as gender, age, weight, and BMI [30]. These variables, along with parity, also correlate with labor pain [24]. In our study, there were no significant differences between the groups with respect to age, weight, height, BMI, gestational week at delivery, and the number of previous $\mathrm{C}$-sections. However, this study has some limitations regarding the research methodology. Firstly, the study included a rela- 
tively small number of patients. Secondly, the preoperative levels of anxiety, a factor known to increase the level of pain felt, were not measured in this study. Given that planned versus emergency C-section may lead to different levels of anxiety in patients, this is a factor that possibly influenced the results. In addition, the fact that we did not control for the hour of delivery may also be a confounding element that was not taken into account in our study as it was suggested to be a possible factor that may affect patients' analgesic intake [25].

\section{Conclusion}

In conclusion, serum leptin levels were higher in patients undergoing C-section with labor pain than in those without labor pain. Increased levels of serum leptin were associated with decreased preoperative pain threshold and increased postoperative analgesic consumption in the whole study group. Postoperative analgesic requirements may vary among patients, and their requirements might be predicted using preoperative indicators. Serum leptin levels could be one such indicator and warrants further studies on a larger sample size.

\section{Statement of Ethics}

This study was approved by the Ethics Committee at Kahramanmaraş Sütçü İmam University; written consent forms were received from all patients. The procedures were in accordance with the ethical standards of the Ethics Committee of Kahramanmaraş Sütçü İmam University and the Helsinki Declaration.

\section{Disclosure Statement}

There are no conflicts of interest.

\section{References}

1 Ahima RS, Qi Y, Singhal NS, Jackson MB, Scherer PE. Brain adipocytokine action and metabolic regulation. Diabetes. 2006 Dec;55 Suppl 2:S145-54.

2 Fantuzzi G, Faggioni R. Leptin in the regulation of immunity, inflammation, and hematopoiesis. J Leukoc Biol. 2000 Oct;68(4):43746.

3 Atawi FA, Warsy AS, Babay Z, Addar M. Leptin concentration during different stages of pregnancy. Clin Exp Obstet Gynecol. 2004; 31(3):211-6.

4 Schubring C, Englaro P, Siebler T, Blum WF, Demirakca T, Kratzsch J, et al. Longitudinal analysis of maternal serum leptin levels during pregnancy, at birth and up to six weeks after birth: relation to body mass index, skinfolds, sex steroids and umbilical cord blood leptin levels. Horm Res. 1998;50(5):276-83.

5 Mise H, Yura S, Itoh H, Nuamah MA, Takemura M, Sagawa N, et al. The relationship between maternal plasma leptin levels and fetal growth restriction. Endocr J. 2007 Dec;54(6): 945-51.

6 Logan CA, Thiel L, Bornemann R, Koenig W, Reister F, Brenner H, et al. Delivery mode, duration of labor, and cord blood adiponectin, leptin, and c-reactive protein: results of the population-based ulm birth cohort studies. PLoS One. 2016 Feb;11(2):e0149918.

7 Yoshimitsu N, Douchi T, Kamio M, Nagata Y. Differences in umbilical venous and arterial leptin levels by mode of delivery. Obstet Gynecol. 2000 Sep;96(3):342-5.
8 Hu ZJ, Han W, Cao CQ, Mao-Ying QL, Mi WL, Wang YQ. Peripheral leptin signaling mediates formalin-induced nociception. Neurosci Bull. 2018 Apr;34(2):321-9.

9 Lim G, Wang S, Zhang Y, Tian Y, Mao J. Spinal leptin contributes to the pathogenesis of neuropathic pain in rodents. J Clin Invest. 2009 Feb;119(2):295-304.

10 Gandhi R, Perruccio AV, Rizek R, Dessouki O, Evans HM, Mahomed NN. Obesity-related adipokines predict patient-reported shoulder pain. Obes Facts. 2013;6(6):536-41.

11 Bedaiwy MA, Falcone T, Goldberg JM, Sharma RK, Nelson DR, Agarwal A. Peritoneal fluid leptin is associated with chronic pelvic pain but not infertility in endometriosis patients. Hum Reprod. 2006 Mar;21(3):788-91.

12 Perruccio AV, Mahomed NN, Chandran V, Gandhi R. Plasma adipokine levels and their association with overall burden of painful joints among individuals with hip and knee osteoarthritis. J Rheumatol. 2014 Feb;41(2): 334-7.

13 Younger J, Kapphahn K, Brennan K, Sullivan SD, Stefanick ML. Association of leptin with body pain in women. J Womens Health (Larchmt). 2016 Jul;25(7):752-60.

14 Klein S, Coppack SW, Mohamed-Ali V, Landt M. Adipose tissue leptin production and plasma leptin kinetics in humans. Diabetes. 1996 Jul;45(7):984-7.
15 EisenachJC,PanPH,SmileyR,Lavand'homme P, Landau R, Houle TT. Severity of acute pain after childbirth, but not type of delivery, predicts persistent pain and postpartum depression. Pain. 2008 Nov; 140(1):87-94.

16 Maeda T, Kiguchi N, Kobayashi Y, Ikuta T, Ozaki M, Kishioka S. Leptin derived from adipocytes in injured peripheral nerves facilitates development of neuropathic pain via macrophage stimulation. Proc Natl Acad Sci USA. 2009 Aug;106(31):13076-81.

17 Rodgers HM, Liban S, Wilson LM. Attenuated pain response of obese mice (B6.Cglep $(o b))$ is affected by aging and leptin but not sex. Physiol Behav. 2014 Jan;123:80-5.

18 Tian Y, Wang S, Ma Y, Lim G, Kim H, Mao J. Leptin enhances NMDA-induced spinal excitation in rats: A functional link between adipocytokine and neuropathic pain. Pain. 2011 Jun;152(6):1263-71.

19 Kutlu S, Canpolat S, Sandal S, Ozcan M, Sarsilmaz M, Kelestimur H. Effects of central and peripheral administration of leptin on pain threshold in rats and mice. Neuro Endocrinol Lett. 2003 Jun-Aug;24(3-4):193-6.

20 Dunbar JC, Lu H. Leptin-induced increase in sympathetic nervous and cardiovascular tone is mediated by proopiomelanocortin (POMC) products. Brain Res Bull. 1999 Oct;50(3):21521.

21 Vrinten DH, Gispen WH, Groen GJ, Adan RA. Antagonism of the melanocortin system reduces cold and mechanical allodynia in mononeuropathic rats. J Neurosci. 2000 Nov; 20(21):8131-7. 
22 Fietta P, Fietta P. Counterbalance between leptin and cortisol may be associated with fibromyalgia. Psychiatry Clin Neurosci. 2006 Aug;60(4):529.

23 Rubino E, Vacca A, Govone F, Gai A, Boschi $\mathrm{S}$, Zucca $\mathrm{M}$, et al. Investigating the role of adipokines in chronic migraine. Cephalalgia. 2017 Oct;37(11):1067-73.

24 Melzack R. Labour pain as a model of acute pain. Pain. 1993 May;53(2):117-20.

25 Carvalho B, Coleman L, Saxena A, Fuller AJ, Riley ET. Analgesic requirements and postoperative recovery after scheduled compared to unplanned cesarean delivery: a retrospective chart review. Int J Obstet Anesth. 2010 Jan; 19(1):10-5.
26 Buhagiar L, Cassar OA, Brincat MP, Buttigieg GG, Inglott AS, Adami MZ, et al. Predictors of post-caesarean section pain and analgesic consumption. J Anaesthesiol Clin Pharmacol. 2011 Apr;27(2):185-91.

27 Ortner CM, Granot M, Richebé P, Cardoso M, Bollag L, Landau R. Preoperative scar hyperalgesia is associated with post-operative pain in women undergoing a repeat Caesarean delivery. Eur J Pain. 2013 Jan;17(1):11123.
28 Pan PH, Coghill R, Houle TT, Seid MH, Lindel WM, Parker RL, et al. Multifactorial preoperative predictors for postcesarean section pain and analgesic requirement. Anesthesiology. 2006 Mar;104(3):417-25.

29 Kirdemir P, Özorak O. Can postoperative pain and analgesic need be predicted in preoperative period? Turkiye Klinikleri J Med Sci. 2011;31(4):951-9.

30 Ostlund RE Jr, Yang JW, Klein S, Gingerich R. Relation between plasma leptin concentration and body fat, gender, diet, age, and metabolic covariates. J Clin Endocrinol Metab. 1996 Nov;81(11):3909-13. 